\title{
Calculation of the Regularized Vacuum Energy in Cavity Field Theories
}

\author{
R. Hofmann \\ Institute of Theoretical Physics, University of Tuebingen, Auf der Morgenstelle 14, \\ 72076 Tuebingen, Germany \\ M. Schumann and R.D. Viollier \\ Institute of Theoretical Physics, University of Cape Town, Rondebosch 7r01, South Africa
}

\begin{abstract}
A novel technique based on Schwinger's proper time method is applied to the Casimir problem of the M.I.T. bag model. Calculations of the regularized vacuum energies of massless scalar, Dirac spinor, and vector fields confined to a static and spherical cavity are presented in a consistent manner. While our results agree with previous calculations based on asymptotic methods, the main advantage of our technique is that the numerical errors are under control. Interpreting the bag constant as a vacuum expectation value, we investigate potential cancellations of boundary divergences between the canonical energy and its bag constant counterpart. It is found that such a cancellation does not occur.
\end{abstract}

PACS numbers: 12.20.DS, 12.39.Ba

Typeset using REVTEX 


\section{INTRODUCTION}

The effects of a classical static background field on the observables of a relativistic quantum field theory have been investigated in detail for quite some time. Perhaps the easiest way of distorting a free quantum field is to impose boundary conditions on a static surface. For the special case of an electromagnetic field, obeying boundary conditions on two parallel, uncharged and static plates, an attractive force was derived by Casimir as early as 1948 [1]. About two decades later, great efforts were made to investigate more complicated geometrical arrangements of the boundaries for a variety of free field theories and boundary conditions [2]. In particular, following the development of the bag models of hadrons [3] [5], there has been increased interest in vacuum energies arising as a consequence of the boundary conditions on a static sphere. More recently, such calculations have been extended to include interacting, renormalizable quantum field theories in the framework of perturbation theory [6].

There are a number of approaches to calculating vacuum expectation values of field operators in relativistic quantum field theories. In general, one can classify these into two categories: local or global methods [2]. The global mode summation techniques avoid the explicit occurrence of divergences by analytical continuation (Zeta function regularization [7], heat kernel expansion [8]), whereas local methods take advantage of the fact that residual local divergences! manifest themselves only as nonintegrable infinities of density functions on the boundary $[9]$.

The main purpose of this paper is to present a consistent calculation of the regularized vacuum energy of massless scalar, Dirac spinor, and vector fields, that are confined to a static and spherical cavity, using the local stress tensor method [9 11. In the framework of the

\footnotetext{
${ }^{1}$ The free-space global divergence which renormalizes the cosmological constant is, in this case, already subtracted. This corresponds to the condition that the vacuum expectation values vanish in the case of a free-space theory.
} 
M.I.T. bag model [3], there is for each field a linear boundary condition that determines the eigenmodes and their energies. There is also a nonlinear boundary condition that guarantees the balance of the pressures at the boundary. The latter is usually taken into account in an average fashion, by minimizing the total energy of the bag with respect to the bag radius. Comparing the result of this minimization with the experimental data, a phenomenological value for the bag constant is obtained. However, the nonlinear boundary conditions of the model can also be used to interpret the bag constant as a vacuum expectation value which makes this additional and rather artificial minimization condition redundant [12].

This paper is organized as follows: In section II, we illustrate the local stress tensor method in the case of the straight-forward example of scalar and Dirac spinor fields satisfying linear boundary conditions on a plane. Well-known results are reproduced using the momentum-space representations of the free-space propagators and the reflection method [13], and introducing Schwinger's spectral $z$-forms [14 21]. Section III contains a derivation of the expressions for the 00-component of the canonical energy-momentum tensor, integrated over the angles, for Klein-Gordon, Dirac, and vector fields satisfying the linear boundary conditions of the MIT bag model on a static, spherical surface. Using the cavity mode representation of the propagators [14, 22, 23], the numerical evaluation is done by summing over the cavity mode quantum numbers first and integrating the resulting spectral $z$-form subsequently. An integration over a regularization volume yields the canonical regularized energy. Thereby an expansion with respect to the regularization parameter is derived from the expansion of the density into a Laurent type series. We apply our technique to cavity quantum chromodynamics (CQCD) 13, 14 to zeroth order in $\alpha_{\mathrm{s}}$. In section IV, the expression for the fermionic bag constant is derived and evaluated in analogy to section III. In the last section, we discuss problems and speculate on their possible solutions, and subsequently, we compare our results with those available in the literature. 


\section{VACUUM ENERGY IN HALF-SPACE FIELD THEORIES}

In this section a method for calculating the vacuum expectation value of the canonical energy density $\theta^{00}$ is illustrated for half-space field theories. Let us consider a massless scalar field subject to Dirichlet (D) and Neumann (N) boundary conditions, and a massless Dirac field satisfying the linear boundary condition of the M.I.T. bag model (MIT,q) [3]

$$
\begin{aligned}
\mathrm{D}: & \left.\phi(x)\right|_{x \in S}=0 \\
\mathrm{~N}: & \left.\partial_{\mu} n^{\mu} \phi(x)\right|_{x \in S}=0 \\
\mathrm{MIT}, \mathrm{q}: & \left.\left(i n_{\mu} \gamma^{\mu}-1\right) \psi(x)\right|_{x \in S}=0, \\
& n:=(0,0,0,1), \quad S:=\left\{x \mid x^{3}=0\right\} .
\end{aligned}
$$

Using the reflection method [13], the half-space propagators read

$$
\begin{aligned}
\Delta_{\mathrm{D}}(x, y) & =\Delta^{0}(x-y)-\Delta^{0}\left(x-y^{T}\right) \\
\Delta_{\mathrm{N}}(x, y) & =\Delta^{0}(x-y)+\Delta^{0}\left(x-y^{T}\right) \\
S_{\mathrm{MIT}, \mathrm{q}}(x, y) & =S^{0}(x-y)-i \gamma^{3} S^{0}\left(x-y^{T}\right), \\
y^{T} & =\left(y^{0}, y^{1}, y^{2},-y^{3}\right),
\end{aligned}
$$

where $S^{0}$ and $\Delta^{0}$ stand for the free-space propagators. The structure of the canonical energymomentum tensor $\theta^{\mu \nu}$ (see for example [24]) requires that, in order to obtain the (diverging) vacuum energy densities, some bilinear operators must be applied to the corresponding propagators. For a massless Klein-Gordon field, we obtain

$$
\left\langle\theta^{00}(x)\right\rangle=\lim _{y \rightarrow x} \partial_{x^{0}} \partial_{y^{0}} \Delta(x, y)
$$

whereas, in the case of a massless Dirac field, the canonical vacuum energy density reads

$$
\left\langle\theta^{00}(x)\right\rangle=\lim _{y \rightarrow x} \frac{i}{2} \operatorname{Tr}\left[\left(\partial_{x^{0}}-\partial_{y^{0}}\right) \gamma^{0}\right] S(x, y)
$$

where $\Delta(x, y)$ and $S(x, y)$ denote the field propagators, respectively. 
Let us now give a general illustration of the $z$-form method for the calculation of the vacuum expectation value of local field bilinears. As an example, we concentrate on the expectation value of a Lorentz scalar in a scalar field theory, characterized by the bilinear $\mathcal{B}$. This scalar quantity can be expressed in terms of the following identity

$$
\langle\mathcal{B}(\phi(x), \phi(x))\rangle=\lim _{y \rightarrow x} \mathcal{B}_{x, y} \Delta^{0}(x-y) .
$$

Here $\Delta^{0}(x-y)$ stands for the free-space Feynman propagator, and $\mathcal{B}_{x, y}$ denotes the point splitted version of $\mathcal{B}$. In momentum space, the propagator is given by

$$
\Delta^{0}(p) \propto \frac{1}{p^{2}}
$$

and the application of $\mathcal{B}$ results in

$$
\mathcal{B}_{x, y} \frac{\mathrm{e}^{-i p(x-y)}}{p^{2}} \propto \frac{b\left(p^{2}\right)}{p^{2}} \mathrm{e}^{-i p(x-y)},
$$

where $b$ is a polynomial in $p^{2}$ (in a scale invariant theory $b$ is just a power in $p^{2}$ corresponding to the mass dimension of $\mathcal{B}(\phi(x), \phi(x)))$. We now take the limit $y \rightarrow x$ and rotate to Euclidean momentum space

$$
p_{0} \rightarrow i p_{0}
$$

The (Euclidean) denominator can then be elevated into an exponent

$$
\frac{1}{p^{2}} \rightarrow \int_{0}^{\infty} d z \mathrm{e}^{-z p^{2}}
$$

and the remaining task is to integrate over the (Euclidean) momentum which involves (modified) Gaussian integrals

$$
\int \frac{d p_{\mu}}{(2 \pi)}\left\{1,\left(p_{\mu}\right)^{2}, \ldots\right\} \mathrm{e}^{-z\left(p_{\mu}\right)^{2}}=\left\{1, \frac{1}{2 z}, \ldots\right\} \frac{1}{\sqrt{4 \pi z}}, \quad \mu=0, \ldots, 3 .
$$

\footnotetext{
${ }^{2}$ The Dirac and vector cases can be treated in straightforward analogy.

${ }^{3}$ A contribution of a product of field derivatives $\partial_{\mu} \phi(x) \partial_{\nu} \phi(x)$ to $\mathcal{B}$, e.g., implies a contribution of $\partial_{x^{\mu}} \partial_{y^{\nu}}$ to $\mathcal{B}_{x, y}$
} 
Calculating $\left\langle\theta^{00}\right\rangle$ for a massless scalar field that is distorted by a Dirichlet plate at $x^{3}=0$ is now straightforward. The full propagator for this problem reads

$$
\begin{aligned}
\Delta(x, y) & =\Delta^{0}(x-y)-\Delta^{0}\left(x-y^{T}\right), \\
y^{T} & =\left(y^{0}, y^{1}, y^{2},-y^{3}\right) .
\end{aligned}
$$

Renormalizing the cosmological constant, by applying the bilinear operator of Eq. (3) to the difference of the half- and free-space propagators, yields finite results in the interior of the cavity. In the case of Dirichlet boundary conditions, we obtain

$$
\begin{aligned}
\left\langle\theta^{00}\left(x^{3}\right)\right\rangle_{D} & =-\frac{i}{(2 \pi)^{4}} \int d^{4} p \frac{\left(p^{0}\right)^{2}}{p^{2}+i 0} \mathrm{e}^{-2 i x^{3} p^{3}} \\
& =\frac{1}{(2 \pi)^{4}} \int_{e} d^{4} p \frac{\left(p^{0}\right)^{2}}{p^{2}} \mathrm{e}^{-2 i x^{3} p^{3}} \\
& =\frac{1}{32 \pi^{2}} \int_{0}^{\infty} d z \mathrm{e}^{-\left(x^{3}\right)^{2} / z} z^{-3} \\
& =\frac{1}{32 \pi^{2}\left(x^{3}\right)^{4}},
\end{aligned}
$$

while for Neumann boundary conditions we have

$$
\left\langle\theta^{00}\left(x^{3}\right)\right\rangle_{N}=-\frac{1}{32 \pi^{2}\left(x^{3}\right)^{4}} .
$$

In the case of a massless Dirac field, subject to the linear boundary condition of the M.I.T. bag model for the quark field (see Eqs. (1)), we obtain

$$
\left\langle\theta^{00}\left(x^{3}\right)\right\rangle_{\mathrm{MIT}, \mathrm{q}} \equiv 0 .
$$

This result is a consequence of the tracelessness of the energy-momentum tensor for this field [ [9].

\section{VACUUM ENERGY IN FIELD THEORIES}

In this section, we shall calculate the canonical energy density of the vacua of massless scalar, Dirac, and vector fields, confined to a static and spherical cavity with radius $R$. 
After a brief description of the methods, the regularized vacuum energy is calculated for each field. We then apply our results to CQCD in zeroth order perturbation theory, i.e. for $\alpha_{\mathrm{S}}=0$ [3, [3].

\section{A. Massless scalar fields}

In order to perform the free-space subtraction in the spherical symmetric case, we need to express the free scalar propagator in terms of angular momentum eigenstates using the Rayleigh expansion for plane waves

$$
\begin{aligned}
\Delta^{0}(x-y)= & -\frac{i}{(2 \pi)^{4}} \int d^{4} p \frac{\mathrm{e}^{-i p(x-y)}}{p^{2}+i 0} \\
= & -(4 \pi)^{2} \frac{i}{(2 \pi)^{4}} \int d^{4} p \frac{1}{p^{2}+i 0} \\
& \times \sum_{\substack{l, l^{\prime} \\
m, m^{\prime}}} i^{l}(-i)^{l^{\prime}} j_{l}(p r) j_{l^{\prime}}\left(p r^{\prime}\right) Y_{l, m}(\hat{r}) Y_{l, m}^{*}(\hat{p}) \\
& \times Y_{l^{\prime}, m^{\prime}}^{*}\left(\hat{r}^{\prime}\right) Y_{l^{\prime}, m^{\prime}}(\hat{p}) .
\end{aligned}
$$

Applying the bilinear operator of Eq. (3) to this representation of the propagator, and performing in turn the angular integration, the summation over $m$ and a Euclidean rotation yields, after introducing the $z$-integration according to Eq. (8),

$$
\left\langle\theta^{00}(r)\right\rangle=\frac{1}{2 \pi^{5 / 2}} \int_{0}^{\infty} d z \int_{0}^{\infty} d k k^{2} \sum_{l}(2 l+1)\left(j_{l}(k r)\right)^{2} \frac{\mathrm{e}^{-z k^{2}}}{z^{3 / 2}} .
$$

For the cavity part, we obtain the vacuum expectation value of the canonical energy density by applying the bilinear operator of Eq. (3) to the cavity mode representation of the propagator (see Appendix A). A $z$-integration is introduced, which originates from a shift of the (Euclidean) momentum squared denominator of the propagator into an exponential, as dis-

cussed in the preceding section. Here the meaning of the term momentum differs somewhat from that of free space due to the boundary conditions, i.e. the analogue to the expression

$$
p^{2}=\left(p^{0}\right)^{2}-(\vec{p})^{2}
$$

in free space, is in the cavity 


$$
\left(p_{n, l}\right)^{2}=\omega^{2}-\left(\varepsilon_{n, l}\right)^{2} .
$$

Here $\omega$ denotes the arbitrary (off-shell) energy, and $\varepsilon_{n, l}$ stands for the energy of the mode, labelled by the radial quantum number $n$ and angular momentum quantum number $l$.

The result of the free-space subtracted canonical vacuum energy density in the cavity is then

$$
\begin{aligned}
\left\langle\tilde{\theta}^{00}(r)\right\rangle_{\mathrm{D}, \mathrm{N}}:= & 4 \pi\left\langle\theta^{00}(r)\right\rangle_{\mathrm{D}, \mathrm{N}} \\
= & -\frac{1}{4 \pi^{\frac{1}{2}}} \int_{0}^{\infty} d z \frac{1}{z^{\frac{3}{2}}} \sum_{l}\left\{\sum_{n, \mu} \int d \Omega\left[a_{n, l, \mu}^{D, N}(\vec{x}) a_{n, l, \mu}^{* \mathrm{D}, \mathrm{N}}(\vec{x})\right] \mathrm{e}^{-z \varepsilon_{n, l}^{2}}\right. \\
& \left.\quad-\frac{2(2 l+1)}{\pi} \int_{0}^{\infty} d k k^{2}\left(j_{l}(k r)\right)^{2} \mathrm{e}^{-z k^{2}}\right\} \\
= & -\frac{1}{4 \pi^{\frac{1}{2}}} \int_{0}^{\infty} d z \frac{1}{z^{\frac{3}{2}}} \sum_{l}(2 l+1)\left\{\sum_{n} \frac{1}{R^{3}} \mathcal{N}_{n, l}^{2 \mathrm{D}, \mathrm{N}}\left(j_{l}\left(\left|\varepsilon_{n, l}\right| r\right)\right)^{2} \mathrm{e}^{-z \varepsilon_{n, l}^{2}}\right. \\
& \left.\quad-\frac{2}{\pi} \int_{0}^{\infty} d k k^{2}\left(j_{l}(k r)\right)^{2} \mathrm{e}^{-z k^{2}}\right\}
\end{aligned}
$$

Here, the $a_{n, l, \mu}^{D, N}(\vec{x})$ denote the scalar cavity modes for either Dirichlet or Neumann boundary conditions, and the $\mathcal{N}_{n, l}^{\mathrm{D}, \mathrm{N}}$ stand for their normalization constants [14,23], respectively, as explained in Appendix A. Using the plane-wave representation of the free-space propagator implies a $z^{-3}$ divergence in the free-space part of Eq. (18). Since finite sums of linearly independent functions cannot change the divergence structure common to all of these terms, a numerical evaluation of $\left\langle\tilde{\theta}^{00}(r)\right\rangle_{\mathrm{D}, \mathrm{N}}$ would diverge.

The calculation reveals a substantial difference between the Dirichlet and the Neumann case. The Dirichlet boundary condition yields a $z$-form that is integrable at $z=0$, whereas the Neumann boundary condition leads to a nonintegrable $z^{-3 / 2}$ divergence. We are able to show that this divergence is a global one, i.e. independent on $r^{\prime}:=r / R$. It therefore resembles another volume divergence (as does the free-space global divergence), and hence it can be omitted through a renormalization of the cosmological constant. 


\section{B. Massless Dirac fields}

Using Eq. (4), the mode summation representing the cavity Dirac propagator [14,23], the spherical representation of the free-space propagator, and introducing the Schwinger $z$-integral, we obtain, after an angular integration,

$$
\begin{aligned}
&\left\langle\tilde{\theta}^{00}(r)\right\rangle_{\mathrm{MIT}, \mathrm{q}}:=4 \pi\left\langle\theta^{00}(r)\right\rangle_{\mathrm{MIT}, \mathrm{q}} \\
&=\frac{1}{2 \pi^{\frac{1}{2}}} \int_{0}^{\infty} d z \frac{1}{z^{\frac{3}{2}}}\left\{\sum_{\kappa} \frac{1}{2} \sum_{n, \mu} \int d \Omega\left[q_{n, \kappa, \mu}^{\dagger}(\vec{x}) q(\vec{x})_{n, \kappa, \mu}\right] \mathrm{e}^{-z \varepsilon_{n, \kappa}^{2}}\right.\left.\quad-\sum_{l} \frac{4}{\pi}(2 l+1) \int_{0}^{\infty} d k k^{2}\left(j_{l}(k r)\right)^{2} \mathrm{e}^{-z k^{2}}\right\} \\
&=\frac{1}{2 \pi^{\frac{1}{2}}} \int_{0}^{\infty} d z \frac{1}{z^{\frac{3}{2}}}\left\{\sum_{\kappa} \frac{1}{2} \sum_{n} \frac{1}{R^{3}} \mathcal{N}_{n, \kappa}^{2}(2 J+1)\left(\left(j_{l}\left(\left|\varepsilon_{n, \kappa}\right| r\right)\right)^{2}+\left(j_{\bar{l}}\left(\left|\varepsilon_{n, \kappa}\right| r\right)\right)^{2}\right) \mathrm{e}^{-z \varepsilon_{n, \kappa}^{2}}\right. \\
&\left.\quad-\sum_{l} \frac{4}{\pi}(2 l+1) \int_{0}^{\infty} d k k^{2}\left(j_{l}(k r)\right)^{2} \mathrm{e}^{-z k^{2}}\right\}
\end{aligned}
$$

where

$$
\begin{aligned}
J & :=|\kappa|-\frac{1}{2}, \\
l & :=|J|+\frac{1}{2} \operatorname{sgn} \kappa, \\
\bar{l} & =l-\operatorname{sgn} \kappa .
\end{aligned}
$$

Here, $q_{n, \kappa, \mu}(\vec{x})$ denotes the Dirac cavity mode, labelled with the radial quantum number $n$, the Dirac quantum number $\kappa$, and the angular momentum projection $\mu$, as discussed in Appendix $\mathrm{A}$.

\section{Massless vector fields}

We restrict ourselves to vector fields satisfying the linear boundary conditions of the MIT bag model as e.g. the gluon fields [3, 13]

$$
\begin{aligned}
\text { MIT,g: } & \left.n_{\mu} F^{\mu \nu}(x)\right|_{x \in S}=0 \\
& \left.n_{\mu} A^{\mu}(x)\right|_{x \in S}=0
\end{aligned}
$$


where $F^{\mu \nu}$ and $n_{\mu}$ are defined as

$$
\begin{aligned}
& F^{\mu \nu}:=\partial^{\mu} A^{\nu}-\partial^{\nu} A^{\mu}, \\
& \left(n_{\mu}\right):=(0, \hat{r}),
\end{aligned}
$$

respectively.

Fixing the gauge in a covariant fashion by means of the Fadeev-Popov procedure and setting the gauge parameter $\lambda$ equal to unity, corresponding to the Feynman gauge, yields the field equation and linear boundary conditions for the various polarizations $\Sigma$ of the vector field

$$
\begin{aligned}
\left(\nabla^{2}+\varepsilon^{2}\right) a^{\mu}= & \\
\left.\hat{r} \cdot \vec{\nabla} a_{\mathcal{S}}^{0}(x)\right|_{x \in S}= & 0, \\
\left.\hat{r} \cdot \vec{a}_{\mathcal{L}}(x)\right|_{x \in S}= & \hat{r} \times\left.\left(\vec{\nabla} \times \vec{a}_{\Sigma}\right)(x)\right|_{x \in S}=0, \\
& \Sigma=\mathcal{L}, \mathcal{M}, \mathcal{E} .
\end{aligned}
$$

Here $\Sigma$ runs over the scalar $(\mathcal{S})$, longitudinal $(\mathcal{L})$, magnetic $(\mathcal{M})$, and electric $(\mathcal{E})$ polarizations [14,23].

Expanding both, the cavity and the free-space propagators, in terms of polarization and applying the bilinear operator

$$
-\lim _{y \rightarrow x} \operatorname{Tr}_{\mu, \nu} \partial_{x^{0}} \partial_{y^{0}}
$$

to their difference yields

$$
\begin{aligned}
&\left\langle\tilde{\theta}^{00}(r)\right\rangle_{\mathrm{MIT}, \mathrm{g}}:=4 \pi\left\langle\theta^{00}(r)\right\rangle_{\mathrm{MIT,g}} \\
&=-\frac{1}{2 \pi^{\frac{1}{2}}} \int_{0}^{\infty} d z \frac{1}{z^{\frac{3}{2}}} \sum_{l} \sum_{n, \rho, \Sigma} \int d \Omega g^{\Sigma \Sigma}\left\{\frac{1}{2} a_{(n, l, \rho, \Sigma)}^{\mu}(\vec{x}) a_{\mu(n, l, \rho, \Sigma)}^{*}(\vec{x}) \mathrm{e}^{-z \varepsilon_{(n, l, \Sigma)}^{2}}\right. \\
&\left.\quad-\frac{1}{\pi} \int_{0}^{\infty} d k k^{2} \tilde{a}_{(k, l, \rho, \Sigma)}^{\mu}(\vec{x}) \tilde{a}_{\mu(k, l, \rho, \Sigma)}^{*}(\vec{x}) \mathrm{e}^{-z k^{2}}\right\} .
\end{aligned}
$$

Here, $g^{\Sigma \Sigma}$ denotes the polarization tensor

$$
\left(g^{\Sigma \Sigma}\right)=\operatorname{diag}(1,-1,-1,-1),
$$


and $a_{(n, l, \rho, \Sigma)}^{\mu}(\vec{x})$ stands for the normalized gluon cavity mode with radial quantum number $n$, total angular momentum $l$, angular projection $\rho$, and polarization $\Sigma$, as detailed in Appendix A. The mode $\tilde{a}_{(k, l, \rho, \Sigma)}^{\mu}$ is a not normalized free-space gluon mode and therefore labelled with a continuous energy $k$. Omitting all quantum labels and hence disregarding the difference between discrete and continuous energies, we can trim our notation and obtain the following relations 14,23

$$
\begin{aligned}
a_{\mathcal{S}}^{0}(\vec{x}) & =\frac{\mathcal{N}_{\mathcal{S}}}{R^{\frac{3}{2}}} i j_{l}(\varepsilon r) Y_{l, \rho}(\hat{r})=: \frac{\mathcal{N}_{\mathcal{S}}}{R^{\frac{3}{2}}} \tilde{a}_{\mathcal{S}}^{0}(\vec{x}) \\
\vec{a}_{\mathcal{L}}(\vec{x}) & =\frac{\mathcal{N}_{\mathcal{L}}}{(2 l+1) R^{\frac{3}{2}}}\left\{\sqrt{l} j_{l-1}(\varepsilon r) \vec{Y}_{l, \rho}^{l-1}(\hat{r})+\sqrt{l+1} j_{l+1}(\varepsilon r) \vec{Y}_{l, \rho}^{l+1}(\hat{r})\right\} \\
& =: \frac{\mathcal{N}_{\mathcal{L}}}{R^{\frac{3}{2}}} \overrightarrow{\tilde{a}}_{\mathcal{L}}(\vec{x}) \\
\vec{a}_{\mathcal{M}}(\vec{x}) & =\frac{\mathcal{N}_{\mathcal{M}}}{R^{\frac{3}{2}}} j_{l}(\varepsilon r) \vec{Y}_{l, \rho}^{l}(\hat{r})=: \frac{\mathcal{N}_{\mathcal{M}}}{R^{\frac{3}{2}}} \overrightarrow{\tilde{a}}_{\mathcal{M}}(\vec{x}) \\
\vec{a}_{\mathcal{E}}(\vec{x}) & =\frac{\mathcal{N}_{\mathcal{E}}}{(2 l+1) R^{\frac{3}{2}}}\left\{\sqrt{l+1} j_{l-1}(\varepsilon r) \vec{Y}_{l, \rho}^{l-1}(\hat{r})-\sqrt{l} j_{l+1}(\varepsilon r) \vec{Y}_{l, \rho}^{l+1}(\hat{r})\right\} \\
& =: \frac{\mathcal{N}_{\mathcal{E}}}{R^{\frac{3}{2}}} \overrightarrow{\tilde{a}}_{\mathcal{L}}(\vec{x}) .
\end{aligned}
$$

Here, $\mathcal{N}_{\Sigma}$ denote cavity normalization constants of the various polarization modes (see Appendix A), and the $\vec{Y}_{l, \rho}^{l \pm 1}(\hat{r})$ stand for vector spherical harmonics [14,23].

\section{Numerical evaluation of $\left\langle\tilde{\theta}^{00}\right\rangle$}

Eqs. (18), (19), and (24) are suitable for a numerical evaluation. The $k$ integration is performed in the range from zero to $\varepsilon_{\max }$, the maximal energy eigenvalue used in the sum over cavity modes. In our computations it is typically of the size $200 / R$.

The calculation of $\left\langle\tilde{\theta}^{00}\right\rangle$ is done in two steps. At first, we compute the $z$-form for the corresponding field and linear boundary condition at a number $M$ of points $r^{\prime}(M \approx 500$ and $r^{\prime}:=r / R$ ). It thereby proves convenient to make the variable substitution $z=y^{2}$ resulting in a pure $y^{-2}$ divergence in the $y$-form. In a second step, we integrate the regular part of the $y$-form and hence determine the energy density as a function of the position $r^{\prime}$. 
In Figs. 1 and 3, the $y$-forms for $\left\langle\tilde{\theta}^{00}\right\rangle$ are displayed for the scalar Dirichlet and the Dirac case. The $y$-forms for the spatial vector polarizations show a similar behavior. It is shown numerically that the region of small $y$, where the form is practically zero, moves to the left with increasing maximal energy $\varepsilon_{\max }$, whereas points to the right of this region remain stable. The $y$-forms for $\left\langle\tilde{\theta}^{00}\right\rangle$ in the scalar Neumann case, or equivalently the vector case with scalar polarization, contain stable (under an increase of $\varepsilon_{\max }$ ) and global, i.e. independent of $r^{\prime}$, $y^{-2}$-divergences (see Fig. 2). The subtraction of these divergences amounts to renormalizing the cosmological constant and results in $y$-forms shown in Fig. 2 .

The error $f\left(r^{\prime}, \varepsilon_{\max }\right)$ of $\left\langle\tilde{\theta}^{00}\right\rangle$ due to the truncation of the sum over cavity energies in Eqs. (18), (19) and (24) can be determined by varying the cut-off energy $\varepsilon_{\max }$. We can safely estimate the upper bound for $f\left(r^{\prime}, \varepsilon_{\max }\right)$ at $10^{-6}$, valid for all $r^{\prime}$ and all fields and boundary conditions under consideration. As an example, Fig. 1 shows $\left\langle\tilde{\theta}^{00}\left(r^{\prime}\right)\right\rangle$ for a Dirichlet scalar field.

Following Deutsch and Candelas [9], we may expand $\left\langle\tilde{\theta}^{00}\right\rangle$ into a Laurent type series around $r^{\prime}=1$

$$
\begin{gathered}
\left\langle\tilde{\theta}^{00}\right\rangle\left(\delta^{\prime}\right)=\frac{1}{R^{4}}\left[c_{-4}\left(\delta^{\prime}\right)^{-4}+c_{-3}\left(\delta^{\prime}\right)^{-3}+\ldots\right. \\
\left.+c_{-1}\left(\delta^{\prime}\right)^{-1}+c_{0}+\ldots+c_{N}\left(r^{\prime}\right)^{N}\right] \\
N>0, \quad \delta^{\prime}:=1-r^{\prime}
\end{gathered}
$$

To extract the coefficients of the negative powers in Eq. (26), the function

$$
E^{L}\left(\delta^{\prime}\right):=c_{-4}\left(\delta^{\prime}\right)^{-4}+\ldots+c_{-1}\left(\delta^{\prime}\right)^{-1}
$$

is fitted to the calculated curve in the interval

$$
\delta_{\min }^{\prime} \leq \delta^{\prime} \leq \delta_{\max }^{\prime}
$$

where $\delta_{\min }^{\prime}$ and $\delta_{\max }^{\prime}$ are close to zero. For the determination of the positive power coefficients in Eq. (26), we fit the polynomial 


$$
E^{T}\left(r^{\prime}\right):=a_{0}+a_{1} r^{\prime}+\ldots+\left(r^{\prime}\right)^{N}
$$

to the calculated curve within the interval

$$
0 \leq r^{\prime} \leq r_{\max }^{\prime}:=1-\delta_{\max }^{\prime}
$$

A value of $N=9$ is used to obtain negligible fitting errors. Fitting $E^{L}\left(\delta^{\prime}\left(r^{\prime}\right)\right)$ to the polynomial

$$
b_{0}+b_{1} r^{\prime}+\cdots+b_{N}\left(r^{\prime}\right)^{N}
$$

within the above interval, the analytical expression for $\left\langle\tilde{\theta}^{00}\right\rangle$ reads

$$
\begin{aligned}
\left\langle\tilde{\theta}^{00}\left(r^{\prime}\right)\right\rangle & =E^{L}\left(\delta^{\prime}\left(r^{\prime}\right)\right)+\Delta E\left(r^{\prime}\right), \\
\Delta E\left(r^{\prime}\right) & :=c_{0}+c_{1} r^{\prime}+\cdots+c_{N}\left(r^{\prime}\right)^{N}, \\
c_{i} & :=a_{i}-b_{i}, \quad(0 \leq i \leq N) .
\end{aligned}
$$

The numerical errors of the coefficients $c_{-4}, \ldots, c_{-1}$ can be estimated by varying the interval

$$
\delta_{\min }^{\prime} \leq \delta^{\prime} \leq \delta_{\max }^{\prime}
$$

In our computations we vary $\delta_{\min }^{\prime}$ from 0.05 to 0.15 and $\delta_{\max }^{\prime}$ from 0.1 to 0.2 to obtain errors of about $1 \%, 10 \%$ and up to $130 \%$ for the coefficients of the leading, next to leading, and the weakest divergences, respectively.

In the literature, there are several attempts to extract boundary divergences of $\left\langle\tilde{\theta}^{00}\right\rangle$ or the canonical energy $E\left(\varepsilon^{\prime}\right)$ using the asymptotic properties of analytic functions [9, 10, 12, 25]. The drawback of these expansions lies in the fact that the errors of the analysis are out of control. In our calculation of the regularized energy, we start with a determination of $\left\langle\tilde{\theta}^{00}\right\rangle$, where the only source of substantial errors is the fitting procedure to the Laurent type series. However, these errors can be estimated and made smaller in more extensive calculations. 


\section{E. The regularized canonical vacuum energy}

Following Bender and Hays [10], we regularize the canonical energy $E$ by integrating $\left\langle\theta^{00}\left(r^{\prime}\right)\right\rangle$ only to an upper limit of

$$
r_{\max }^{\prime}=\left(1-\varepsilon^{\prime}\right), \quad \varepsilon^{\prime}:=\frac{\varepsilon}{R},
$$

where $\varepsilon$ denotes the distance from the boundary. Using Eq. (26), we obtain

$$
\begin{aligned}
& E\left(\varepsilon^{\prime}\right)= \frac{1}{R} \int_{0}^{1-\varepsilon^{\prime}} d r^{\prime} r^{\prime 2}\left\langle\tilde{\theta}^{00}\left(r^{\prime}\right)\right\rangle \\
&=\frac{1}{R}\left[\tilde{c}_{-3} \varepsilon^{\prime}{ }^{-3}+\tilde{c}_{-2} \varepsilon^{\prime-2}+\tilde{c}_{-1} \varepsilon^{\prime-1}\right. \\
& \\
&\left.\quad+\tilde{c}_{\log } \log \varepsilon^{\prime}+\tilde{c}_{0}+\mathcal{O}\left(\varepsilon^{\prime}\right)\right] .
\end{aligned}
$$

The coefficients $\{c\}$ of Eq. (26) and the coefficients $\{\tilde{c}\}$ of Eq. (28) are related by

$$
\begin{aligned}
\tilde{c}_{-3} & =\frac{1}{3} c_{-4}, & \tilde{c}_{-2} & =-c_{-4}+\frac{1}{2} c_{-3}, \\
\tilde{c}_{-1} & =c_{-4}-2 c_{-3}+c_{-2}, & \tilde{c}_{\text {log }} & =-c_{-3}+2 c_{-2}-c_{-1},
\end{aligned}
$$

for the divergent terms, whereas the coefficients of the positive powers of $\varepsilon^{\prime}$ depend also on the truncation number $N$. Since we are only interested in the limit $\varepsilon^{\prime} \rightarrow 0$, the only substantial coefficient of $\left\{\tilde{c}_{j} \mid j \geq 0\right\}$ is $\tilde{c}_{0}$, given by

$$
\tilde{c}_{0}=-\frac{1}{3} c_{-4}+\frac{3}{2} c_{-3}-\frac{3}{2} c_{-1}+\sum_{i=0}^{N} \frac{1}{i+3} c_{i} .
$$

For the fermionic case, we assume $c_{-4}=0$ since here the canonical energy-momentum tensor coincides with the improved tensor, and hence it is traceless [9]. Table \ contains a list of the coefficients $\{\tilde{c}\}$ for the various fields and boundary conditions, where the errors are determined from the errors of the coefficients $\{c\}$ according to the rules of error propagation. Note that the leading divergences for the scalar Dirichlet and Neumann fields exhibit a behavior analogous to that of the half-space problem. 


\section{F. Cavity quantum chromodynamics to zeroth order in $\alpha_{\mathrm{s}}$}

We are now in a position to calculate the regularized vacuum energy for CQCD [3, 13. without interaction of quarks and gluons. The expression for $\left\langle\theta^{00}\right\rangle_{\mathrm{CQCD}}$ in a covariantly gauge-fixed theory can be found, for example, in Ref. [24]. Choosing the Feynman gauge and taking into account $S U(2)$ flavour symmetry with massless up- and down-quarks, we obtain

$$
\left\langle\theta^{00}\right\rangle_{\mathrm{CQCD}}=6 \times\left\langle\theta^{00}\right\rangle_{\mathrm{MIT}, \mathrm{q}}+8 \times\left(\left\langle\theta^{00}\right\rangle_{\mathrm{MIT}, \mathrm{g}}-2 \times\left\langle\theta^{00}\right\rangle_{\mathrm{N}}\right)
$$

The scalar Neumann part resembles the contribution of the unphysical ghost fields [22]. Table 1 contains the set of coefficients $\{\tilde{c}\}$ that we obtain for the expansion of $E_{\mathrm{CQCD}}\left(\varepsilon^{\prime}\right)$ corresponding to Eq. (28).

\section{THE FERMIONIC BAG CONSTANT}

As explained in Ref. [3], the bag constant $B$ is introduced into the Lagrangian to achieve conservation of a Poincaré generator, i.e. the energy. In bag model calculations $B$ is determined using the minimization condition

$$
\frac{d}{d R} E(R)=0
$$

where $E(R)$ denotes the total energy of the bag. Milton 12 suggests that one should calculate the bag constant from first principles, by interpreting it as a vacuum expectation value. The nonlinear boundary condition of the model then serves as a definition for $B$. Here we will carry out the calculation of this vacuum expectation value for a massless fermion field, subject to the boundary conditions of the M.I.T. bag model on a static sphere with radius $R$. The expression for $B_{\mathrm{MIT}, \mathrm{q}}$ reads

$$
B_{\mathrm{MIT}, \mathrm{q}}:=-\left.\frac{1}{2}\left\langle\partial_{r}(\bar{\psi} \psi)\right\rangle\right|_{r=R}
$$

and implies that the differential operator 


$$
-\lim _{y \rightarrow x} \frac{1}{2} \operatorname{Tr}\left(\partial_{r_{x}}+\partial_{r_{y}}\right)
$$

should be applied to the difference of the cavity and the free-space propagator. We expect $B_{\mathrm{MIT}, \mathrm{q}}$ to be infinite. In order to regularize it, we compute it at some interior point a distance $\varepsilon$ away from the boundary, rather than at some point on the boundary, as Eq. (32) demands. For the angular integrated version $\tilde{B}_{\mathrm{MIT}, \mathrm{q}}\left(\varepsilon^{\prime}\right)$ of $B_{\mathrm{MIT}, \mathrm{q}}\left(\varepsilon^{\prime}\right)$, we arrive at

$$
\begin{aligned}
\tilde{B}_{\mathrm{MIT}, \mathrm{q}}\left(\varepsilon^{\prime}\right):= & 4 \pi B_{\mathrm{MIT}, \mathrm{q}}\left(\varepsilon^{\prime}\right) \\
= & -\frac{1}{\pi^{1 / 2}} \int_{0}^{\infty} d z \frac{1}{z^{1 / 2}} \sum_{\kappa}(2 J+1) \\
& \times \sum_{n>0} \mathcal{N}_{n, \kappa}^{2} \varepsilon_{n, \kappa}^{2}\left\{\frac{j_{l}\left(\left|\varepsilon_{n, \kappa}\right| r\right)}{2 l+1}\left(l j_{l-1}\left(\left|\varepsilon_{n, \kappa}\right| r\right)-(l+1) j_{l+1}\left(\left|\varepsilon_{n, \kappa}\right| r\right)\right)\right. \\
& \left.\quad-\frac{j_{\bar{l}}\left(\left|\varepsilon_{n, \kappa}\right| r\right)}{2 \bar{l}+1}\left(\bar{l} j_{\bar{l}-1}\left(\left|\varepsilon_{n, \kappa}\right| r\right)-(\bar{l}+1) j_{\bar{l}+1}\left(\left|\varepsilon_{n, \kappa}\right| r\right)\right)\right\} \mathrm{e}^{-z \varepsilon_{n, \kappa}^{2}}, \\
\varepsilon^{\prime}= & \frac{\varepsilon}{R}, \quad r^{\prime}:=1-\varepsilon^{\prime} .
\end{aligned}
$$

There is no free-space part in Eq. (34) since $\operatorname{Tr} \gamma^{\mu} \equiv 0$ for $\mu=0, \ldots, 3$.

The result of the calculation of the coefficients $\{\tilde{c}\}$ in the expansion of the regularized bag constant energy $E_{\mathrm{MIT}, \mathrm{q}}^{B}\left(\varepsilon^{\prime}\right)$ with

$$
\begin{aligned}
E_{\mathrm{MIT}, \mathrm{q}}^{B}\left(\varepsilon^{\prime}\right):= & \frac{R^{3}}{3}\left(1-\varepsilon^{\prime}\right)^{3} \times \tilde{B}_{\mathrm{MIT}, \mathrm{q}}\left(\varepsilon^{\prime}\right) \\
= & \frac{1}{R}\left[\tilde{c}_{-4} \varepsilon^{\prime-4}+\tilde{c}_{-3} \varepsilon^{\prime-3}+\tilde{c}_{-2} \varepsilon^{\prime-2}\right. \\
& \left.\quad+\tilde{c}_{-1} \varepsilon^{\prime-1}+\tilde{c}_{0}+\mathcal{O}\left(\varepsilon^{\prime}\right)\right]
\end{aligned}
$$

is displayed in Table III. Here we also list the set of coefficients in the expansion of the total vacuum energy $E_{\mathrm{MIT}, \mathrm{q}}^{\mathrm{tot}}\left(\varepsilon^{\prime}\right)$ given by

$$
\begin{aligned}
E_{\mathrm{MIT}, \mathrm{q}}^{\mathrm{tot}}\left(\varepsilon^{\prime}\right) & :=\int_{0}^{1-\varepsilon^{\prime}} d r^{\prime}\left(r^{\prime}\right)^{2}\left\{\left\langle\tilde{\theta}^{00}\left(r^{\prime}\right)\right\rangle_{\mathrm{MIT}, \mathrm{q}}-\tilde{B}_{\mathrm{MIT}, \mathrm{q}}\left(\varepsilon^{\prime}\right)\right\} \\
& =E_{\mathrm{MIT}, \mathrm{q}}\left(\varepsilon^{\prime}\right)-E_{\mathrm{MIT}, \mathrm{q}}^{B}\left(\varepsilon^{\prime}\right) .
\end{aligned}
$$




\section{SUMMARY AND DISCUSSION}

The main purpose of this paper was to investigate the effect of a static and spherical boundary on the canonical vacuum energy densities of otherwise free massless Klein-Gordon, Dirac, and vector fields. Thereby the linear boundary condition of the M.I.T. bag model [3] has been used. A Green's function method, that is based on the eigenmode representation of the propagator and the Schwinger parametrization of the Euclidean momentum squared denominator, has been used to obtain numerical results for the vacuum energy densities. For the fermionic field, a calculation of the regularized bag constant based on its definition via the nonlinear boundary condition was carried out. Numerical results for the densities have been fitted to Laurent type series in powers of the distance to the boundary. The expressions obtained in this manner could be integrated within a regularization volume to yield an expansion of the energies in terms of the regularization parameter. Numerical errors of the coefficients in these expansions turn out to be less than $1 \%$ for the leading singularity, and up to $100 \%$ for the weakest divergences. In general, the finite part $\tilde{c}_{0}$ could be determined to about $50 \%$ accuracy, using this method.

We can compare our results directly with those in the literature. Bender and Hays 10 have calculated the leading divergence of the canonical part of the vacuum energy using a Green's function method. They introduced their regularization in the same fashion as we do, but they relied on analytical formulae for the asymptotic expansion of Bessel functions, without error estimate. For example in the fermionic and the Dirichlet scalar case, the comparison is as follows:

$$
\begin{aligned}
& \text { Bender \& Hays : } \tilde{c}_{-3}^{\mathrm{MIT}, \mathrm{q}}=-\frac{1}{120 \pi} \approx-0.0027, \quad \tilde{c}_{-4}^{\mathrm{D}}=-\frac{1}{24 \pi} \approx-0.0133 \\
& \text { this work : } \quad \tilde{c}_{-3}^{\mathrm{MIT}, \mathrm{q}}=-0.01058 \pm 0.45 \%, \quad \tilde{c}_{-4}^{\mathrm{D}}=0.01324 \pm 0.1 \%
\end{aligned}
$$

There are, of course, disagreements, but the sign error of Bender and Hays' result for the

scalar case has already been pointed out by Milton [12]. The factor four difference in the fermion case might be due to an omission of the trace as required by Eq. (4). 
Olaussen and Ravndal [25] used a Green's function method to calculate the electromagnetic canonical vacuum energy density for a spherical bag, and we are thus not able to compare their results with ours. Milton [12] calculated the canonical fermionic vacuum energy using a different regularization than ours which again leaves no possibility of comparison.

The small error of the leading infinite terms allows us to compare the results for the canonical and the bag constant part in the fermionic case. A cancellation of the infinities between these two contributions to the total energy does not occur, since there is a nonvanishing quartic divergence in the bag constant contribution, whereas the leading infinite term in the canonical part is quadratic (see Table 【). There is also a logarithmic divergence in the canonical part which cannot be cancelled by the bag constant contribution. Besides, the coefficient $\tilde{c}_{0}$ in $E_{\mathrm{MIT}, \mathrm{q}}^{B}$ is quite large.

One may speculate that, in a gauge theory calculated perturbatively, infinities stemming from the canonical and the bag constant part of the energy should cancel each other leaving a meaningful finite part. We hope to investigate this matter in the near future. If there is still no hint for a cancellation, one would argue that the introduction of a constant bag energy density $B$ is a too naive device to achieve the conservation of the Poincaré generator, i.e. the energy. Perhaps, a locally conserved energy-momentum tensor (if at all definable on physical grounds) would reveal finite vacuum energies. On the other hand, using the canonical energy-momentum tensor together with soft boundaries, i.e. practically confining potentials (as for example a harmonic oscillator potential), could possibly bypass the global vacuum infinities. The drawbacks of this method are the enormous numerical effort and the necessity of a cutoff parameter to ensure confinement. Moreover, it is quite unsatisfactory, that this additional parameter would have to be determined experimentally. Along the same lines, there have been suggestions to introduce phenomenological parameters in the expression for the bag energy. These parameters could absorb the divergences. However, they should be determined experimentally [6, 12]. A model that needs this (artificial) procedure can surely not be regarded as fundamental.

A possible extension of the work done here would be a more extensive calculation (deter- 
mination of the energy density at points closer to the boundary) to achieve higher precision for the coefficients of the nonleading divergences. Also a calculation of the first order in $\alpha_{\mathbf{s}}$ correction to the free vacuum energy of CQCD could in principle be carried out. One then would encounter problems like the renormalization in the cavity [14] and the non-diagonality of self-energies in the radial quantum number.

\section{ACKNOWLEDGMENTS}

One of us (R.H.) would like to express his gratitude for the warm hospitality extended to him at the Physics Department of the University of Cape Town. Financial contributions from the Foundation for Fundamental Research (FFR) and the Graduiertenkolleg "Struktur und Wechselwirkung von Hadronen und Kernen" are gratefully acknowledged.

\section{APPENDIX A:}

Here, the eigenmodes and propagators for massless Dirac, vector, and scalar fields in a static spherical cavity of radius $R$ are given [22, 23,26].

\section{Massless fermion fields}

The cavity modes for massless fermion fields satisfying the linear boundary condition of the M.I.T. bag model are given by the Dirac spinors

$$
q_{n, \kappa, \mu}(\vec{x})=\left(\begin{array}{c}
g_{n, \kappa}(r) \chi_{\kappa}^{\mu}(\hat{r}) \\
i f_{n, \kappa}(r) \chi_{-\kappa}^{\mu}(\hat{r})
\end{array}\right)
$$

where $\chi_{\kappa}^{\mu}(\hat{r})$ is the usual two-component spherical spinor. Here $n, \kappa$, and $\mu$ denote the radial, Dirac, and magnetic quantum numbers respectively, and the radial functions $g_{n, \kappa}(r)$ and $f_{n, \kappa}(r)$ are given in terms of the spherical Bessel functions $j_{l}$ by

$$
\begin{aligned}
g_{n, \kappa}(r) & =\frac{\mathcal{N}_{n, \kappa}}{R^{3 / 2}} j_{l}\left(p_{n, \kappa} r\right) \\
f_{n, \kappa}(r) & =\frac{\mathcal{N}_{n, \kappa} \operatorname{sgn}(n) \operatorname{sgn}(\kappa)}{R^{3 / 2}} j_{\bar{l}}\left(p_{n, \kappa} r\right) .
\end{aligned}
$$


The discrete momenta $p_{n, \kappa}$ in Eqs. (A2) are determined by the linear boundary condition

$$
\left.(i \vec{\gamma} \cdot \hat{r}+1) q_{n, \kappa, \mu}(\vec{r})\right|_{r=R}=0
$$

which leads to the eigenvalue equation

$$
j_{l}\left(x_{n, \kappa}\right)+\operatorname{sgn}(n) \operatorname{sgn}(\kappa) j_{\bar{l}}\left(x_{n, \kappa}\right)=0 .
$$

The normalization constant $\mathcal{N}_{n, \kappa}$ in Eqs. (A2) is given by

$$
\mathcal{N}_{n, \kappa}=\left(2 \omega_{n}\left(\omega_{n}+\kappa\right)\right)^{-1 / 2}\left|\frac{x_{n}}{j_{l}\left(x_{n}\right)}\right| \text {. }
$$

Here we have introduced the dimensionless energy and momentum parameters

$$
\begin{aligned}
x_{n, \kappa} & =p_{n, \kappa} R, \\
\omega_{n, \kappa} & =\operatorname{sgn}(n) x_{n, \kappa} .
\end{aligned}
$$

The cavity propagator for massless fermions can be represented in terms of these cavity modes as

$$
S\left(x, x^{\prime}\right)=i \sum_{\kappa \nu \mu} q_{n, \kappa, \mu}(\vec{x}) \bar{q}_{n, \kappa, \mu}\left(\vec{x}^{\prime}\right) \int \frac{d \omega}{2 \pi} \frac{e^{-i \omega\left(x_{0}-x_{0}^{\prime}\right)}}{\omega-\varepsilon_{n} \pm i 0},
$$

where the usual Feynman prescription for the poles is used.

\section{Massless vector fields}

In the Feynman gauge, the cavity modes of the massless vector fields are solutions of the d'Alembert equation subject to the linear M.I.T. boundary conditions, as indicated in Eq. (22). The eigenmodes are given in terms of spherical Bessel functions, spherical harmonics, and vector spherical harmonics as

$$
\begin{aligned}
a_{m \mathcal{S}}^{0}(\vec{x}) & =\frac{\mathcal{N}_{m \mathcal{S}}}{R^{3 / 2}} i j_{J}\left(\varepsilon_{m}^{\mathcal{S}} r\right) Y_{J M}(\hat{r}), \\
\vec{a}_{m \mathcal{L}}(\vec{x}) & =\frac{\mathcal{N}_{m \mathcal{L}}}{\sqrt{R^{3}(2 J+1)}}\left[\sqrt{J} j_{J-1}\left(\varepsilon_{m}^{\mathcal{L}} r\right) \vec{Y}_{J M}^{J-1}(\hat{r})+\sqrt{J+1} j_{J+1}\left(\varepsilon_{m}^{\mathcal{L}} r\right) \vec{Y}_{J M}^{J+1}(\hat{r})\right], \\
\vec{a}_{m \mathcal{M}}(\vec{x}) & =\frac{\mathcal{N}_{m \mathcal{M}}}{R^{3 / 2} j_{J}\left(\varepsilon_{m}^{\mathcal{M}} r\right) \vec{Y}_{J M}^{J}(\hat{r}),} \\
\vec{a}_{m \mathcal{E}}(\vec{x}) & =\frac{\mathcal{N}_{m \mathcal{E}}}{\sqrt{R^{3}(2 J+1)}}\left[\sqrt{J+1} j_{J-1}\left(\varepsilon_{m}^{\mathcal{E}} r\right) \vec{Y}_{J M}^{J-1}(\hat{r})-\sqrt{J} j_{J+1}\left(\varepsilon_{m}^{\mathcal{E}} r\right) \vec{Y}_{J M}^{J+1}(\hat{r})\right],
\end{aligned}
$$


where $m$ denotes the set of quantum numbers $\{N, J, M\}$ (radial, angular momentum, and magnetic respectively). The energy $\varepsilon_{m}^{\Sigma}$ of the mode with polarization $\Sigma$ is determined from the eigenvalue conditions

$$
\begin{aligned}
J j_{J}\left(\varepsilon_{m}^{\Sigma} R\right)-\varepsilon_{m}^{\Sigma} R j_{J+1}\left(\varepsilon_{m}^{\Sigma} R\right) & =0, \\
\Sigma=\mathcal{S}, \mathcal{L} & \\
(J+1) j_{J}\left(\varepsilon_{m}^{\mathcal{M}} R\right)-\varepsilon_{m}^{\mathcal{M}} R j_{J+1}\left(\varepsilon_{m}^{\mathcal{M}} R\right) & =0, \\
j_{J}\left(\varepsilon_{m}^{\mathcal{E}} R\right) & =0 .
\end{aligned}
$$

Here $\mathcal{S}$ denotes the scalar, $\mathcal{L}$ the longitudinal, $\mathcal{M}$ the transverse magnetic, and $\mathcal{E}$ the transverse electric polarization [23]. The normalization constants appearing in Eqs. (A8) are

$$
\begin{aligned}
\mathcal{N}_{m \Sigma}=\sqrt{\frac{2}{\left(\varepsilon_{m}^{\Sigma} R\right)^{2}-J(J+1)}}\left|\frac{\varepsilon_{m}^{\Sigma} R}{j_{J}\left(\varepsilon_{m}^{\Sigma} R\right)}\right|, \\
\Sigma=\mathcal{S}, \mathcal{L}, \mathcal{M} \\
\mathcal{N}_{m \mathcal{E}}=\frac{\sqrt{2}}{\left|j_{J+1}\left(\varepsilon_{m}^{\mathcal{E} R} R\right)\right|} .
\end{aligned}
$$

A representation of the cavity propagator for massless vector fields in terms of the eigenmodes is

$$
D^{\mu \nu}\left(x, x^{\prime}\right)=-i \sum_{m \Sigma} g^{\Sigma \Sigma} a_{m \Sigma}^{\mu}(\vec{x}) a_{m \Sigma}^{\nu *}\left(\vec{x}^{\prime}\right) \int \frac{d \omega}{2 \pi} \frac{e^{-i \omega\left(x_{0}-x_{0}^{\prime}\right)}}{\omega^{2}-\left(\varepsilon_{m}^{\Sigma}\right)^{2}+i 0},
$$

where $g^{\Sigma \Sigma^{\prime}}$ denotes the metric tensor in the polarization space

$$
\begin{gathered}
g^{\mathcal{S S}}=-g^{\mathcal{L} L}=-g^{\mathcal{M M}}=-g^{\mathcal{E}}=1 \\
g^{\Sigma \Sigma^{\prime}}=0 \quad \text { if } \quad \Sigma \neq \Sigma^{\prime}
\end{gathered}
$$

\section{Massless scalar fields}

The cavity modes of the massless scalar fields are the same as the modes of the scalar polarization of the vector fields, i.e. 


$$
\phi_{n, l, \mu}(\vec{x})=\frac{\mathcal{N}_{n, l}^{\mathrm{D}, \mathrm{N}}}{R^{3 / 2}} i j_{l}\left(\varepsilon_{n, l} r\right) Y_{l \mu}(\hat{r}) .
$$

A mode representation of the propagator reads as

$$
\Delta\left(x, x^{\prime}\right)=i \sum_{n, l, \mu} \phi_{n, l, \mu}(\vec{x}) \phi_{n, l, \mu}^{*}\left(\vec{x}^{\prime}\right) \int \frac{d \omega}{2 \pi} \frac{e^{-i \omega\left(x_{0}-x_{0}^{\prime}\right)}}{\omega^{2}-\left(\varepsilon_{n, l}\right)^{2}+i 0} .
$$

The energy eigenvalues $\varepsilon_{n, l}$ depend, of course, on the chosen boundary condition. In the Dirichlet case, we have the eigenvalue equation

$$
j_{l}\left(\varepsilon_{n, l} R\right)=0
$$

which is the same as for the electric polarization of the vector fields. The only difference is that we have to take into account the solutions for $l=0$ which are just $\varepsilon_{n, 0}=n \pi$.

In the case of Neumann boundary conditions, the eigenvalue equation is

$$
l j_{l}\left(\varepsilon_{n, l} R\right)-\varepsilon_{n, l} R j_{l+1}\left(\varepsilon_{n, l} R\right)=0
$$

which yields the same spectrum as for the scalar polarization of the vector fields. The normalization constant $\mathcal{N}_{n, l}^{\mathrm{N}}$ is given by Eq. (A10a and $\mathcal{N}_{n, l}^{\mathrm{D}}$ by Eq. (A10b). 


\section{REFERENCES}

[1] H. B. G. Casimir, Proc. K. Ned. Akad. Wet. 51, 793 (1948).

[2] G. Plunien, B. Müller, and W. Greiner, Phys. Rep. 134, 89 (1986).

[3] A. Chodos et al., Phys. Rev. D 9, 3471 (1974).

[4] W. A. Bardeen et al., Phys. Rev. D 11, 1094 (1975).

[5] T. D. Lee, Phys. Rev. D 19, 1802 (1979).

[6] M. Bordag, D. Robaschik, and E. Wieczorek, Ann. Phys. 165, 192 (1985).

[7] V. V. Nesterenko and I. G. Pirozhenko, Phys. Rev. D 57, 1284 (1998).

[8] M. Bordag, E. Elizalde, and K. Kirsten, J. Math. Phys. 37, 895 (1996).

[9] D. Deutsch and P. Candelas, Phys. Rev. D 20, 3063 (1979).

[10] C. M. Bender and P. Hays, Phys. Rev. D 14, 2622 (1976).

[11] K. A. Milton, L. DeRaad, and J. Schwinger, Ann. Phys. 115, 388 (1978).

[12] K. A. Milton, Phys. Rev. D 22, 1444 (1980).

[13] T. H. Hansson and R. L. Jaffe, Phys. Rev. D 28, 882 (1983).

[14] A. J. Stoddart, Ph.D. thesis, University of Cape Town, 1989.

[15] G. U. Schreiber and R. D. Viollier, Ann. Phys. 215, 277 (1992).

[16] G. U. Schreiber and R. D. Viollier, Phys. Lett. B 279, 131 (1992).

[17] A. J. Stoddart and R. D. Viollier, Nuc. Phys. A 532, 657 (1991).

[18] A. J. Stoddart and R. D. Viollier, Nuc. Phys. A 541, 623 (1992).

[19] P. R. Page, R. J. Lindebaum, and R. D. Viollier, Nuc. Phys. A 506, 1003 (1993).

[20] J. A. Cuthbert and R. D. Viollier, Z. Phys. C 58, 295 (1993). 
[21] R. D. Viollier, Prog. Part. Nucl. Phys. 36, 251 (1996).

[22] R. F. Buser, R. D. Viollier, and P. Zimak, Int. J. Theor. Phys. 27, 925 (1988).

[23] M. S. O’Connor and R. D. Viollier, Ann. Phys. 248, 268 (1996).

[24] P. Pascual and R. Tarrach, QCD: Renormalization for the practitioner (Springer Verlag, Heidelberg, 1984).

[25] K. Olaussen and F. Ravndal, Nuc. Phys. B 192, 237 (1981).

[26] R. D. Viollier, S. A. Chin, and A. K. Kerman, Nuc. Phys. A 407, 269 (1983). 


\section{TABLES}

TABLE I. The coefficients $\{\tilde{c}\}$ for the divergent and the finite parts of the canonical vacuum energy $E\left(\varepsilon^{\prime}\right)$ for massless Dirchlet (D) and Neumann (N) scalar, fermion (MIT,q) and vector fields (MIT,g), and CQCD (CQCD) to zeroth order.

\begin{tabular}{cccccc}
\hline \hline & $\tilde{c}_{-3}$ & $\tilde{c}_{-2}$ & $\tilde{c}_{-1}$ & $\tilde{c}_{\log }$ & $\tilde{c}_{0}$ \\
\hline $\mathrm{D}$ & $0.0132435 \pm 0.1 \%$ & $-0.0232 \pm 10 \%$ & $-0.0065 \pm 40 \%$ & $-0.0052 \pm 10 \%$ & $-10.2 \pm 50 \%$ \\
$\mathrm{~N}$ & $-0.01318 \pm 0.5 \%$ & $-0.0012 \pm 60 \%$ & $-0.085 \pm 40 \%$ & $0.64 \pm 15 \%$ & $45 \pm 60 \%$ \\
$\mathrm{MIT}, \mathrm{q}$ & 0 & $-0.01058 \pm 0.45 \%$ & $-0.35 \pm 100 \%$ & $-0.73 \pm 100 \%$ & $0.16 \pm 20 \%$ \\
$\mathrm{MIT}, \mathrm{g}$ & $0.24 \pm 7 \%$ & $-1.6 \pm 50 \%$ & $27 \pm 50 \%$ & $120 \pm 55 \%$ & $85 \pm 90 \%$ \\
$\mathrm{CQCD}$ & $0.23 \pm 7.5 \%$ & $-1.1 \pm 70 \%$ & $20 \pm 90 \%$ & $80 \pm 50 \%$ & $72 \pm 80 \%$ \\
\hline \hline
\end{tabular}

TABLE II. The coefficients $\{\tilde{c}\}$ for the divergent and the finite part of $E_{\mathrm{MIT}, \mathrm{q}}^{B}$ and the total vacuum energy $E_{\mathrm{MIT}, \mathrm{q}}^{\mathrm{tot}}\left(\varepsilon^{\prime}\right)$.

\begin{tabular}{lcccccc}
\hline \hline & $\tilde{c}_{-4}$ & $\tilde{c}_{-3}$ & $\tilde{c}_{-2}$ & $\tilde{c}_{-1}$ & $\tilde{c}_{\log }$ & $\tilde{c}_{0}$ \\
\hline$E_{\mathrm{MIT}, \mathrm{q}}^{B}$ & $-0.1593 \pm 0.2 \%$ & $0.375 \pm 1 \%$ & $-0.119 \pm 5 \%$ & $0.11 \pm 20 \%$ & 0 & $-7.2 \times 10^{5} \pm 50 \%$ \\
$E_{\mathrm{MIT}, \mathrm{q}}^{\mathrm{tot}}$ & $0.1593 \pm 0.2 \%$ & $-0.375 \pm 1 \%$ & $0.23 \pm 6 \%$ & $-0.46 \pm 85 \%$ & $-0.7 \pm 100 \%$ & $7.2 \times 10^{5} \pm 50 \%$ \\
\hline \hline
\end{tabular}




\section{FIGURES}

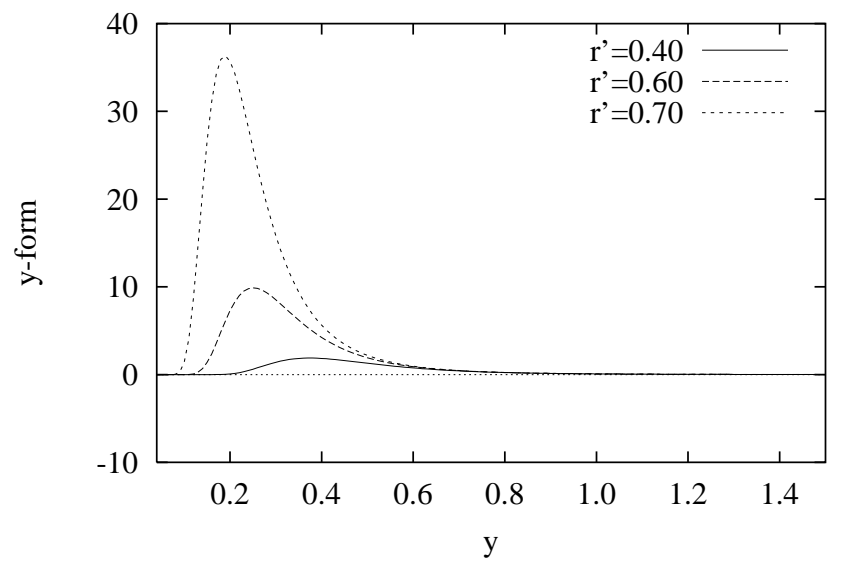

FIG. 1. $y$-forms for $\left\langle\theta^{00}\right\rangle$ at three different locations $r^{\prime}=r / R$ for a massless scalar field fulfilling Dirichlet boundary conditions on a static sphere with radius $R$.

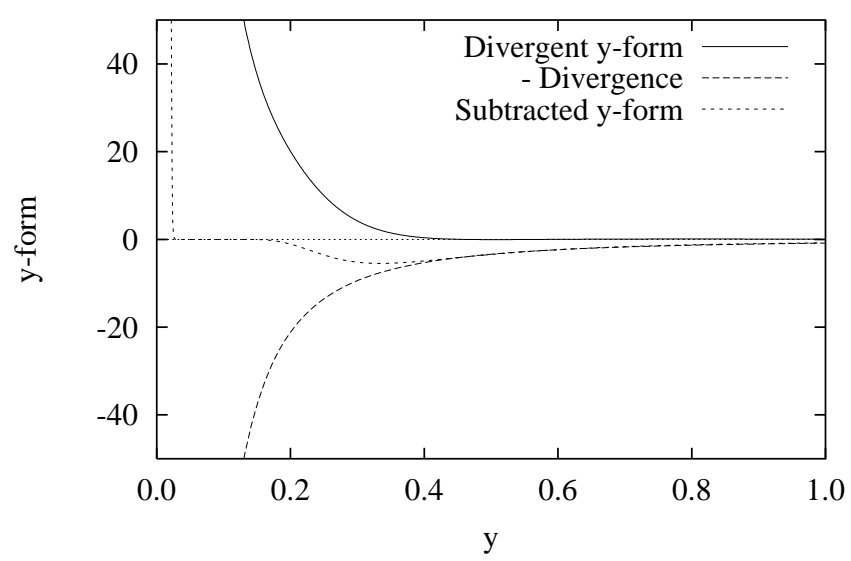

FIG. 2. Unsubtracted and subtracted $y$-forms for $\left\langle\theta^{00}\right\rangle$ at $r^{\prime}=0.5$ for a massless scalar field fulfilling Neumann boundary conditions on a static sphere with radius $R$. The subtracted divergence is of the form $m / y^{2}$, where $m=0.8463$. 


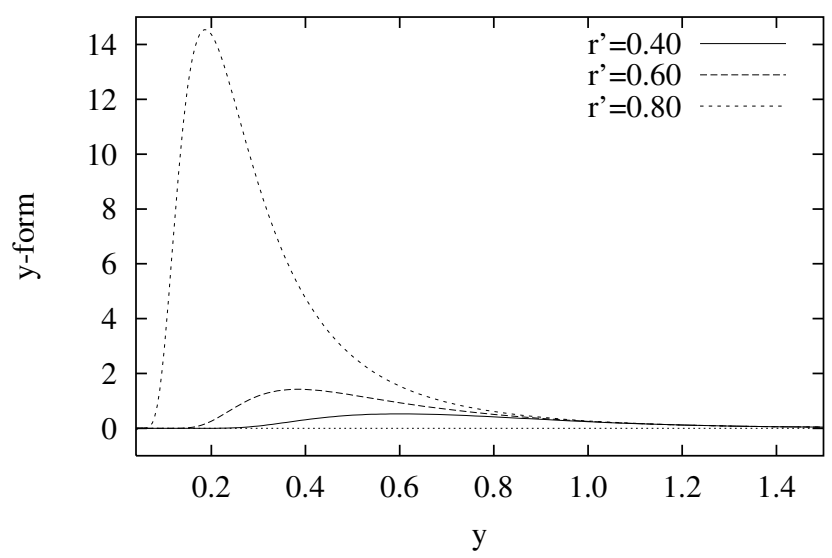

FIG. 3. Massless fermion field $y$-forms at three different locations $r^{\prime}=r / R$. The field modes fulfill the linear boundary condition of the M.I.T. bag model on a static sphere with radius $R$.

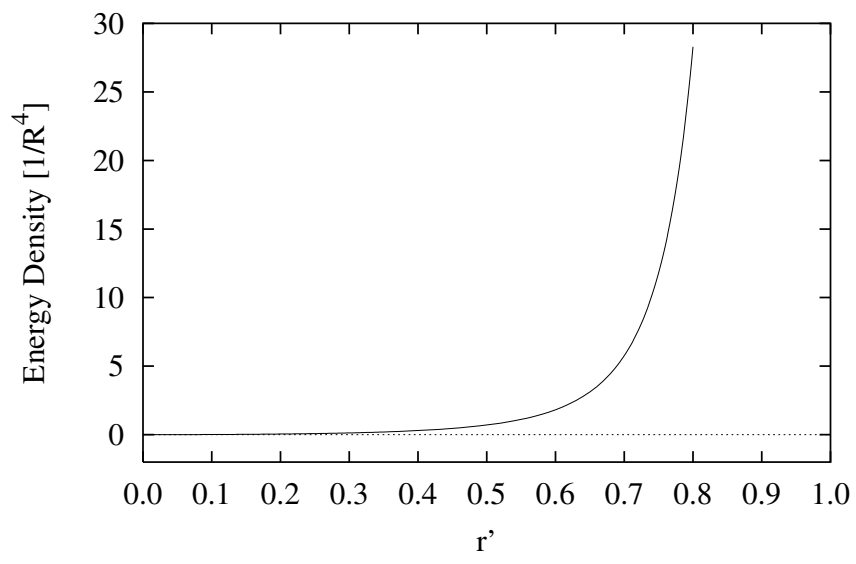

FIG. 4. Vacuum energy density for a massless scalar field fulfilling Dirichlet boundary conditions on a static sphere with radius $R$. 